\title{
Un admirateur de Blaise Pascal : le professeur Reijer Hooykaas
}

Jean-Claude Parlebas

\section{(2) OpenEdition}

1 Journals

Electronic version

URL: http://journals.openedition.org/ccibp/1188

DOI: 10.4000/ccibp.1188

ISSN: 2493-7460

\section{Publisher}

Centre international Blaise Pascal

\section{Printed version}

Date of publication: 1 January 2016

ISBN: 978-2-84516-758-2

ISSN: 0249-6674

\section{Electronic reference}

Jean-Claude Parlebas, « Un admirateur de Blaise Pascal : le professeur Reijer Hooykaas », Courrier du Centre international Blaise Pascal [Online], 38 | 2016, Online since 18 December 2019, connection on 31 March 2020. URL : http://journals.openedition.org/ccibp/1188 ; DOI : https://doi.org/10.4000/ccibp. 1188

This text was automatically generated on 31 March 2020.

Centre international Blaise Pascal 


\title{
Un admirateur de Blaise Pascal : le professeur Reijer Hooykaas
}

\author{
Jean-Claude Parlebas
}

Parmi de très nombreuses études pascaliennes à l'étranger, celle du défunt professeur Reijer Hooykaas a l'avantage d'offrir un point de vue très documenté sur l'apport original et diversifié de Pascal. Dans le présent article, j'aimerais tout d'abord donner quelques éléments sur la personne d'Hooykaas, sur son œuvre en général, puis rendre compte de ses écrits sur Blaise Pascal en particulier, enfin tenter d'expliquer les raisons de son intérêt pour l'œuvre et la vie de Pascal.

\section{Introduction}

2 S'il est couramment admis que Blaise Pascal a été à la fois un scientifique de renom et un penseur chrétien, doté d'une foi personnelle, tout n'a pas été dit sur le sujet. Il vaut donc la peine de continuer à être attentif aux divers travaux, nationaux ou internationaux, centrés sur lui et ses contributions majeures, tant à la science moderne qu'à l'apologétique chrétienne. L'étude sur Pascal du défunt professeur néerlandais Reijer Hooykaas, peu connu du public francophone, a l'avantage d'offrir un point de vue très documenté sur l'apport original et diversifié de Pascal. Ce point de vue me semble encore tout à fait actuel de nos jours. Dans le présent article, j'aimerais tout d'abord donner quelques éléments sur la vie d'Hooykaas qui a été, lui aussi, à la fois scientifique et penseur. Ensuite, je résumerai son œuvre en général et je rendrai compte de ses écrits sur Blaise Pascal en particulier. Finalement, j'essaierai de comprendre les raisons de son intérêt pour l'œuvre et la vie de Pascal qui sont, comme on le sait, intimement liées. 


\section{Quelques éléments sur la personne d'Hooykaas et sur son œuvre en général}

3 Nous donnons ci-dessous une très courte biographie de Reijer Hooykaas, puis nous présentons succinctement divers travaux qu'il a laissés dans la littérature.

\section{Courte biographie}

4 Reijer Hooykaas (1906-1994) a été l'un des premiers universitaires de l'histoire des sciences dans son pays. De plus, comme nous allons le montrer, il n'a pas craint de faire le lien entre science et foi, contribuant ainsi à l'apologétique chrétienne. Il est né dans une famille calviniste d'orfèvres à Schoonhoven (Pays-Bas). Il a d'abord fait des études de chimie et de physique à l'Université d'Utrecht et y a obtenu sa licence en 1933. Il est ensuite devenu professeur de chimie dans l'enseignement secondaire, tout en préparant sa thèse de doctorat. En même temps, il a commencé à publier certains articles concernant la corrélation entre l'histoire des sciences et la pensée religieuse. Ceci lui a valu à terme d'être remarqué par ses collègues (ref [1] \& ref [2]).

En 1946, il est le premier diplômé (doctorat) des Pays-Bas à obtenir une chaire d'histoire des sciences à l'Université Libre d'Amsterdam. A cette époque-là et dans cette Université, on demande à tous les étudiants des sciences de la nature, et même des mathématiques, de suivre les cours de Hooykaas. Plus tard, il a continué d'enseigner l'histoire des sciences à l'Université d'Utrecht, et cela, depuis l'année 1967, jusqu'à sa retraite en 1976. Ensuite, Hooykaas s'est adonné à ses activités d'écrivain, de penseur et de conférencier. Durant de nombreuses années, il a œuvré au sein de la Société Chrétienne des Scientifiques et Médecins aux Pays-Bas. Il a souvent été invité comme orateur par l'association des Chrétiens en Science du Royaume Uni et, à ce titre, il est aussi intervenu aux États-Unis.

\section{Bibliographie générale}

6 En plus de sa parfaite maîtrise de l'anglais et de beaucoup d'autres langues (néerlandais, bien sûr, mais aussi allemand, latin, polonais et portugais) Reijer Hooykaas est à la fois francophone et francophile. J'en veux pour preuve, entre autres, une conférence qu'il a donnée au Palais de la Découverte de notre capitale, dans les années cinquante[3], ainsi qu'une publication sur Pierre de la Ramée (1515-1572)[4]. Une bonne partie de son œuvre résulte d'une analyse faite à partir de la foi chrétienne réformée dont il était convaincu (ref. [5] à ref. [7] ). Dans le même ordre d'idées, il est arrivé à Reijer Hooykaas de s'emporter intérieurement à l'encontre de quelques historiens. Ceux-là prétendaient en effet que certains pionniers de la science moderne devaient forcément avoir des déficiences mentales, vu leurs fortes convictions religieuses. Au nombre de ces pionniers, Hooykaas s'est fait le défenseur, entre autres, de Blaise Pascal, Robert Boyle et Isaac Newton.

Dans son livre: Religion and the Rise of Modern Science[6], c'est-à-dire La religion et la montée de la science moderne, Hooykaas utilise une métaphore: " tandis que les ingrédients du corps de la science ont pu être grecs, ses vitamines et ses hormones étaient bibliques ", affirmant par là l'apport indéniable de la foi judéo-chrétienne à la science de l'Occident. Il a aussi montré comment l'état d'esprit du protestantisme 
naissant, essentiellement sous l'influence de Calvin, a servi au développement de la science moderne.

Il s'est également demandé pourquoi et comment des savants réformés comme Kepler, Boyle, Newton... ont cru devoir s'adonner à la science. Hooykaas nous en donne trois motivations :

1. Pour la gloire de Dieu et au bénéfice de l'humanité.

2. De façon empirique et malgré les idées reçues.

3. En utilisant leurs mains, donc en recourant à l'expérimentation.

9 Cet état d'esprit et cette attitude sont évidemment très proches de ce qu'a vécu et pensé Blaise Pascal lui-même, comme nous le redirons plus loin. À une époque qui est la nôtre, où beaucoup de personnes pensent que la science et la religion sont des ennemis irréductibles, ce livre phare de Hooykaas[6] a le mérite de présenter un autre point de vue, à savoir : la science moderne est, pour une bonne part, un produit de l'influence du judéo-christianisme sur la pensée occidentale.

\section{Écrits de Hooykaas sur Blaise Pascal}

10 S'il y a bien "une sorte de héros » qui ressort de l'œuvre de Reijer Hooykaas, c'est assurément Blaise Pascal avec son essai d'unifier la science et la foi chrétienne. Ce travail de Hooykaas a été publié dès 1939, dans un article, écrit d'abord en néerlandais[8] puis traduit en anglais : ref [9] \& ref [10]. L'objectif de cette publication a été de montrer que les pensées scientifique et religieuse de Pascal forment en fait un tout, quoique Pascal lui-même les ait rigoureusement placées à des niveaux différents ! En fait, la science pascalienne, plus que toute autre science de la même époque, peut être dite «science chrétienne ». Hooykaas nous explique que les attentes de Pascal visà-vis de la science sont tout-à-fait modestes: Pascal est conscient que les mathématiques et la physique sont simplement des activités humaines, et comme telles, elles ne peuvent pas donner lieu à des espérances exagérées! Cette façon pascalienne de concevoir la science est fortement d'inspiration chrétienne. Dans le même ordre d'idées, Pascal a souligné que les sciences historiques et théologiques sont, quant à elles, fondées sur des autorités qui nous ont précédées, en particulier en ce qui concerne la Révélation par les Écritures pour la théologie. Il en va différemment des mathématiques et de la physique qui sont des sciences, connues par la raison, avec, dans le cas de la physique, un apport nécessaire dû à l'expérimentation. Nous rappelons succinctement ci-après comment Pascal considérait les mathématiques, toujours selon l'analyse qu'en fait Hooykaas[9]. C'est à dessein que nous avons repris ci-dessous les mêmes titres, adoptés par cet auteur pour plusieurs développements[9], qui nous ont parus significatifs, mais que nous avons beaucoup résumés.

\section{Mathématiques et limites des mathématiques}

$11 \mathrm{Au}$ sens strict, le terme géométrie est une branche des mathématiques qui traite des points, lignes, angles, surfaces et solides. Cependant Pascal utilise le concept de géométrie pour plusieurs branches des mathématiques: non seulement la géométrie proprement dite, mais aussi l'arithmétique et la mécanique. Voici ce qu'a relevé Hooykaas : 
La meilleure méthode pour trouver la vérité, selon Pascal, est la méthode géométrique. Pourtant, ce n'est pas encore la méthode idéale! Et de poursuivre avec cette méthode idéale : "La vraie méthode...serait de définir tous les termes utilisés et de prouver toutes les propositions avancées. " Mais une telle méthode est impraticable, car «ce qui transcende la géométrie nous dépasse ». Définir et prouver toutes choses est "absolument impossible », toujours selon Pascal, parce que les concepts fondamentaux sur lesquels est fondé le raisonnement humain sont inexplicables[9].

Dans la pensée pascalienne, la raison joue un rôle clef en mathématiques. Pourtant, même cette science, essentiellement logique, atteint, elle aussi, ses limites et se trouve confrontée à des concepts de base indéfinissables. « Par exemple, la divisibilité à l'infini pose problème à l'échelle humaine et l'homme tend à rejeter ce qui est incompréhensible. Mais dans ses Pensées, Pascal a souligné que tout ce qui est incompréhensible ne cesse pas d'exister pour autant ${ }^{9}$. 》

13 Avec Hooykaas, nous constatons que :

même quand Pascal est dans des dispositions d'esprit tout-à-fait rationnelles, il montre finalement aussi son caractère empirique et réaliste. En effet, selon Pascal, la raison humaine ne peut pas vraiment comprendre l'infini, mais nier l'infini conduirait à l'absurde. Ainsi, l'infini est-il au-dessus de la raison, mais le nier est contre la raison[9].

14 En réalité, comme nous le précise Hooykaas :

Pascal a une très haute idée de la «raison ». Il ne la rabaisse que lorsqu'elle s'érige en autorité absolue pour elle-même. Il y a trois causes qui contribuent ensemble à notre connaissance : notre expérience sensorielle, notre raison et le siège de notre cœur, mais la raison n'a pas la suprématie. Pour Pascal, la réalité concrète est toujours supérieure à la raison abstraite...Il est un des pionniers à défendre l'incontournable réalité, face à la méthode de l'abstraction scientifique que Descartes a mise en valeur...Pascal, au contraire, réintègre la validité de l'expérience immédiate, avec son interprétation, à côté de la conception abstraite et purement intellectuelle du monde[9].

15 Ceci est particulièrement vrai en ce qui concerne la physique expérimentale. À ce sujet, faut-il le rappeler, Pascal est l'un des tous premiers savants, à la fois théoricien et expérimentateur. Dans les paragraphes suivants, nous continuons à nous référer brièvement à quelques citations pascaliennes au travers l'œuvre de Hooykaas[9]. C'est aussi une façon de voir ce qui a le plus marqué Hooykaas dans l'analyse qu'il a faite des écrits de notre grand penseur français.

\section{Contribution de Pascal à la physique}

16 Nous rappelons d'abord très brièvement une fameuse controverse scientifique qu'a connue Pascal dans la première moitié du XVII ${ }^{e}$ siècle, puis nous citons Hooykaas à ce sujet. Un exemple typique d'une nouvelle approche de la science, et par conséquent une des premières contributions à la science moderne sont donnés par les expériences de Pascal sur le vide.

17 Celles-ci ont définitivement confirmé et complété les résultats du physicien italien Torricelli.

18 Il faut se souvenir que du temps de Pascal, il existait une interprétation pseudoscientifique, répandue, mais erronée, comme quoi «la nature a horreur de vide». Même Descartes niait l'existence du vide. Quand Torricelli a publié ses premiers 
résultats sur ce thème, Descartes a prétendu qu'une certaine « matière subtile » restait présente dans le tube utilisé pour cette expérience, ce qui revenait à maintenir l'impossibilité du vide!

Soulignons que c'est à ce moment qu'intervient Pascal en proposant de résoudre cette controverse, non par une argumentation rationnelle, mais uniquement par des expériences complémentaires. Pour Pascal, la physique est d'ailleurs essentiellement une science empirique. Voici ce qu'en rapporte Hooykaas :

En physique, les expériences ont un meilleur pouvoir de persuasion que le pur raisonnement...Ce sont les expériences qui montrent la voie à suivre en physique[9].

Hooykaas précise encore :

Des lettrés du Moyen-Âge et également Descartes ont bien affirmé formellement que l'expérience était décisive. Mais, dans leur cas, on peut souvent s'apercevoir que des idées préconçues ont obscurci la pure et bonne interprétation de l'expérience en question. Au contraire, Pascal s'en tient à sa position empirique stricte et est un exemple en la matière[9].

Ainsi, comme il est bien connu, Pascal a définitivement prouvé l'existence du vide. Hooykaas nous rapporte la définition pascalienne de ce vide :

Par ce mot vide, dit Pascal, je désigne toujours un espace qui est vide de tous objets sensibles[9].

Et aussi :

Cette expérience (réalisée par Pascal en escaladant le sommet du Puy-de-Dôme) a révélé que l'eau monte dans le tube en question à des hauteurs différentes qui varient en fonction des déplacements dans le temps, et ceci proportionnellement au poids de l'air...l'effet étant proportionnel à la cause[9].

Hooykaas nous rappelle en outre que Pascal a été le premier à faire le lien entre différents fluides, gazeux ou liquides. Il cite ces paroles du savant qu'il admire :

Pour expliquer sereinement quelles sont les conséquences du poids de l'air, je montre quelles sont les conséquences équivalentes quand il s'agit du poids de l'eau[9].

Soulignons aussi cette remarque importante de Hooykaas :

D'une part, Pascal donne à l'usage du raisonnement toute la place qu'il mérite, mais d'autre part, il insiste pour que ce raisonnement s'en tienne aux exigences d'une stricte logique. Pascal n'emploie aucun terme sans l'avoir bien défini au préalable[9].

Il nous faut mentionner un dernier aspect qui a retenu l'attention d'Hooykaas. Je m'explique : Parfois, les données empiriques recueillies sur une question posée n'ont pas été suffisantes, selon Pascal, pour permettre une conclusion définitive, mais, toute autorité, venue d'ailleurs est d'office disqualifiée pour légiférer en la matière. Blaise Pascal s'est non seulement opposé à toute autorité venue de l'Antiquité, mais aussi à celle issue de la hiérarchie ecclésiastique. De ce point de vue, même une bulle pontificale peut être source de confusion. Voilà pourquoi Pascal s'adresse ainsi aux Jésuites de son temps :

C'est en vain que vous avez obtenu à l'encontre de Galilée ce décret romain qui condamne son opinion sur le mouvement de la terre. Ce n'est pas ce décret qui prouvera que la terre est fixe. De plus, si des observations ultérieures arrivaient à prouver que c'est la terre qui tourne (autour du soleil), même si tous les hommes se mettaient ensemble, ils n'empêcheraient pas qu'elle tourne, ni qu'eux-mêmes tournent avec elle[9]. 

époque, est tout-à-fait moderne!

\section{La Physique et les limites de la science empirique}

Hooykaas nous rapporte l'aversion grandissante de Pascal concernant l'idée ci-après, à savoir que la Raison serait a priori capable d'édifier une science empirique :

Les notes de Pascal intitulées Vanité des sciences ainsi qu'une lettre sur la folie de la science de l'homme nous apportent la preuve qu'il avait l'intention de s'exprimer sur les prétentions d'une fausse science. Et cela probablement dans son Apologie de la Religion Chrétienne[9].

Pour Pascal, Descartes était un cas d'école pour expliquer jusqu'où la Raison peut aller afin de fournir des arguments plausibles sur tout et n'importe quoi. Mais des arguments non vérifiables! Hooykaas précise ceci :

La nièce de Blaise Pascal, Marguerite Perier, écrit à ce sujet que Pascal ne pouvait pas supporter cette façon d'expliquer comment toute chose se forme. Il disait assez fréquemment: «Je n'arrive pas à pardonner à Descartes la chose suivante : C'est qu'il aurait pu facilement imaginer toute sa philosophie, sans faire intervenir Dieu »... Pascal a qualifié le système de Descartes de "Roman de la Nature » et Descartes lui-même de "Docteur de la Raison »...En fait, le seul but de Pascal et d'observer le monde tel qu'il est, et d'établir autant de lois possibles pour décrire comment il est régi... Par conséquent, l'esprit humain va toujours considérer l'exploration de la nature comme une tâche inachevée. La nature avec ses variétés infiniment riches ne nous laisse aucun repos. L'esprit humain se lassera de concevoir, avant que la nature ne cesse d'offrir de nouvelles facettes à explorer. Pour Pascal, la première chose à faire, dans les sciences, c'est d'accepter les données expérimentales, même si parfois elles paraissent incompréhensibles. Or, comme déjà cité plus haut, ce qui est incompréhensible ne cesse pas d'exister pour autant : C'est la ferme conviction de Pascal[9].

\section{Pourquoi Hooykaas s'est-il intéressé à l'œuvre et à la vie de Pascal ?}

Je vais essayer d'énumérer plusieurs raisons.

La première est que Hooykaas est aussi un scientifique (physique-chimie), doublé d'un homme de lettres. De plus, il est un croyant personnellement convaincu, même s'il n'est pas forcément passé par une expérience de conversion subite, comme ce fut le cas pour Pascal. À ces titres, il a pu facilement s'identifier à Blaise Pascal. Nous avons déjà signalé plus haut sa francophonie, voire sa francophilie. Les Pays-Bas ont été historiquement une terre de refuge pour les Huguenots français qui ont fui la Révocation de l'Edit de Nantes à la fin du XVII siècle. Ils ont donc aussi été une terre d'accueil pour la culture française et pour une certaine dissidence catholique (Jansénisme, vieux-Catholicisme).

J'évoquerais une deuxième raison. C'est toujours contraignant de renfermer de grands esprits dans des «boîtes particulières ». Cependant, pour des besoins de clarifications, on peut dire que Reijer Hooykaas se rattache au protestantisme calviniste, tandis que Blaise Pascal s'apparente au catholicisme janséniste. Même si, à l'époque de Pascal, les Jansénistes tenaient à se démarquer des Réformés, probablement pour des raisons 
autant politiques que spirituelles, il est indéniable qu'il y a eu des influences tacites de la foi calviniste vers le Jansénisme. Rappelons aussi que la famille Arnauld, colonne vertébrale du jansénisme français au $\mathrm{xvII}^{\mathrm{e}}$ siècle, vient d'une origine réformée, avant d'avoir plus tard adhéré à la doctrine de l'évêque d'Ypres (Pays Bas) : Cornélius Jansen. Entre minorités, qu'elles soient calviniste ou janséniste, il y a forcément certaines affinités.

Je donnerais une troisième raison qui rapproche beaucoup Hooykaas de Pascal, c'est leur commun recul critique par rapport à certaines formes de leur «religion d'origine ». Je citerais ces mots de Hooykaas qui s'en prend à

la dégénérescence de la théologie réformée, de vivante qu'elle était en un dogmatisme sec et rationaliste, et, après la réaction piétiste contre ce dessèchement de la religion, la dégénérescence du même piétisme en une technique de conversion[4].

Je pense que Blaise Pascal pourrait tout-à-fait souscrire à une conclusion que tire Hooykaas :

En un mot, l'esprit scolastique, la tendance à la sclérose, est général et n'est pas inhérent au caractère de la philosophie scolastique proprement dite. Il est partout où l'esprit qui affranchit et donne la vie est étouffé par la lettre qui tue. C'est une leçon de l'histoire[4]

Une quatrième raison. En tant que scientifique chrétien, Hooykaas lui-même était imprégné de la pensée suivante : La vérité et le respect de la réalité environnante (dont nous faisons partie) sont les deux éléments qui nous motivent dans notre travail scientifique et qui nous servent de garde-fou dans toutes nos frêles prétentions et spéculations humaines. Cette humilité et ce respect n'auraient certainement pas déplu à Blaise Pascal! Je me rends compte à ce propos que Blaise Pascal et Reijer Hooykaas reconnaissent un même cadre général pour leur entreprise de recherche scientifique.

Cinquième raison. Hooykaas, comme Pascal, avait horreur de tout ce qui ressemblait à une construction idéologique, qu'il s'agissait de dogmes cléricaux ou de différents systèmes philosophiques. C'est ainsi que Hooykaas aimait citer cette pensée de Pascal: «Le cœur a ses raisons que la raison ne connaît pas. » Ceci est une manière de remettre la raison humaine à sa place, ainsi que toutes les constructions idéologiques qui en découlent.

Sixième raison. En ce qui concerne le lien entre science et foi judéo-chrétienne, je pense que Hooykaas se sentait proche de Pascal. Voici la boutade qu'a écrite Hooykaas et qui est rapportée dans un article en sa mémoire, in memoriam[11].

S'il nous fallait essayer de construire une science "chrétienne", nous ressemblerions à un homme à la recherche de ses lunettes, mais qui les a déjà sur le nez! La science moderne et la technologie sont en grande partie les fruits du christianisme...Les fondateurs de la science moderne se sont efforcés de trouver une séparation méthodologique de la science et de la religion. Avec Kepler, un chrétien remarquable, l'astronomie a pris son indépendance par rapport aux textes bibliques, mais les notions métaphysiques sont toujours présentes dans sa méthode. Avec Pascal et Boyle, deux apologètes du Christianisme, la séparation science religion devient complète. Dans leurs œuvres respectives, on ne trouve pas un mot de religion, même si leur méthode strictement rationnelle et empirique forme un tout avec leur foi chrétienne...L'histoire des sciences nous démontre clairement que ce qui caractérisait l'œuvre de Pascal, de Boyle, de Newton, de Faraday, etc., c'était le respect des faits empiriques ainsi que de l'indépendance par rapport à la 
théologie et la philosophie. Par conséquent ces scientifiques ont apporté des fruits

durables, contrairement aux spéculateurs et faiseurs de systèmes idéologiques. depuis le début de ma carrière de recherche, une authentique source d'inspiration ainsi qu'un encouragement en ce qui concerne l'apologétique évangélique. Dans la préface de mon doctorat d'Etat, j'ai tenu à écrire ces quelques mots : « En hommage à la grande figure de Blaise Pascal, homme de foi et de génie »[16]. Je suis très fier qu'un esprit aussi brillant que Reijer Hooykaas se soit ainsi passionné pour Blaise Pascal, et cela tout en étant a priori extérieur à notre culture française ! 


\section{BIBLIOGRAPHY}

1. OSTER M., The British Journal for the History of Science (1999) Vol. 32, №3, 366-368

2. COHEN H.F., The Scientific Revolution: Science and Religion in the English Speaking World, Chicago, University of Chicago Press, 2001, p. 61.

3. HOOYKAAS R., La naissance de la cristallographie en France au XVIII ${ }^{e}$ siècle, Les conférences du Palais de la Découverte, Université de Paris, 1953, Série D, №21.

4. HOOYKAAS R., Humanisme, science et Réforme: Pierre de la Ramée, Leyde, E. J. Brille ed., 1958, p. 123.

5. HOOYKAAS R., “Science and Reformation”, Journal of World History, 1956, 3, p. 109-139.

6. HOOYKAAS R., Religion and the Rise of Modern Science, Vancouver, Regent College Publishing, 2000, $162 \mathrm{p}$.

7. HOOYKAAS R., "The Christian Approach in Teaching Science”, Science \& Christian Belief, 1994, 6, p. 113-128.

8. HOOYKAAS R., "Pascal: His science and his religion", Orgaan van de Christelijke Vereeniging van Natuur-en Geneeskundigen in Netherlands, 1939, p. 147-178.

9. HOOYKAAS R., “Pascal: His science and his religion”, Free University Quarterly, 1952, 2,

p. 106-137.

10. Hooykaas R., “Pascal: His science and his religion”, Dutch Classics Tractrix, 1991, p. 115-139.

11. LeEgWATER A., "Reijer Hooykaas: A Modern Advocate for Philosophia Liberia", Perspectives on Science and Christian Faith, 1996, 6, p. 96-105.

12. JAEGER L., Lois de la nature et raisons du cœur. Les convictions religieuses dans le débat épistémologique contemporain, Bern, Peter Lang, coll. « Publications Universitaires Européennes » 2007, 360 p.

13. PARLEBAS J.-C., "Science and Christian Faith in the France of Pascal and Today", Perspectives on Science and Christian Faith, 2015, 67, p. 223-228.

14. PARLEBAS J.C., «Quelles conditions pour une science en bonne santé » in JAEGER L. (dir.) La science peut-elle être neutre?, Lognes, Éd. Farel, Lydia Jaeger, 2010, p. 43-55.

15. PATIER X., Blaise Pascal : La nuit de l'extase, Paris, Les Éditions du Cerf, 2014, 175 pages.

16. PARLEBAS J.C., Effets des interactions entre impuretés sur la structure électronique des métaux de transition, Thèse de Doctorat, Université de Strasbourg, 1976, 192 pages.

\section{AUTHOR}

\section{JEAN-CLAUDE PARLEBAS}

Jean-Claude Parlebas est maintenant Directeur de Recherche honoraire du CNRS. Il était autrefois rattaché à l'Institut de Physique et Chimie des Matériaux de Strasbourg. Il a aussi été récemment l'un des vice-présidents de l'Association des Groupes Bibliques Universitaires de France (AGBUF). 